\title{
Mixed lag synchronization between two complex dynamical networks via hybrid feedback control
}

\author{
Xiaoyun Tang, Zhanying Yang* \\ School of Mathematics and Statistics, South-Central University for Nationalities, Wuhan, Hubei 430074, \\ China
}

*Corresponding author, e-mail: yangzhanying8011@163.com

Received 23 Jun 2018

Accepted 20 Sep 2018

\begin{abstract}
This paper is concerned with mixed lag synchronization between two complex networks, which means that different state variables of the corresponding nodes can attain lag synchronization, antilag synchronization and even amplitude death simultaneously for a given channel time-delay. By using the Lyapunov stability theory and the LaSalle's invariance principle, several sufficient conditions for realizing the mixed lag synchronization between two networks are obtained with two kinds of schemes based on pinning feedback control. Finally, some numerical simulations are provided to demonstrate the effectiveness and feasibility of the results.
\end{abstract}

KEYWORDS: complex network, mixed outer synchronization, pinning control

MSC2010: 34D06, 93B52

\section{INTRODUCTION}

As an important and typical dynamic behavior of complex networks, synchronization has attracted considerable attention of many researchers from various fields. Meanwhile, many kinds of synchronization have been proposed, such as complete synchronization, lag synchronization, projective synchronization, phase synchronization, cluster synchronization, and generalized synchronization. In many real situations, complex networks can not generally synchronize by their own coupling. As a result, some control schemes are developed to design controllers, such as feedback control, adaptive control, impulsive control, intermittent control, and pinning control ${ }^{1}$. Pinning control is a very effective control scheme whose goal is to control the whole network by exerting controllers to a part of nodes. For a large scale network, priority is always given to pinning control due to its advantages, such as simplifying the coupling topology configuration and saving control cost ${ }^{2}$.

In the last decade, outer synchronization has attracted much attention due to its extensive applications in ecology, sociology, and communication, etc. Outer synchronization between two coupled complex networks means that the corresponding nodes of coupled networks will achieve synchronization regardless of synchronization within each network $^{3}$. We refer the readers to Refs. 3-6 and the references therein, where some outer synchronization phenomena have been studied, such as complete outer synchronization, inverse outer synchronization, and other kinds of generalized outer synchronization. Mixed outer synchronization ${ }^{7}$ is a recently developed generalized synchronization conception, which means that different state variables of the corresponding nodes can arrive at complete synchronization, antisynchronization, and even amplitude death simultaneously. This kind of synchronization is called mixed synchronization in Ref. 8, where mixed synchronization between the drive and the response systems can be realized by designing a matrix type unidirectional openplus-closed-loop coupling. It was pointed out in Ref. 8 that mixed synchronization is very useful in practical applications. For example, in processing industry, it is necessary to control the concentration of all reacting chemical constituents in different proportions. Based on mixed synchronization, the concentration of any constituent in a reaction system can either be reduced or enhanced, and it even can be made to be zero. In neuron oscillators, in order to obtain a desired neural dynamics, any state variable can be switched off as membrane voltage while other membrane current can be reduced by using mixed synchronization. On the other hand, mixed outer synchronization can be also regarded as a kind of hybrid projective synchronization ${ }^{9,10}$, in which 
the drive and response systems are synchronized up to a scaling matrix. As we know, hybrid projective synchronization is the development of projective synchronization, which was first studied in Mainieri and Rehacek ${ }^{11}$. Nowadays, hybrid projective synchronization has been extensively studied ${ }^{12,13}$ since it can be used to achieve faster communication ${ }^{14-16}$ and enhance the security in secure communication ${ }^{10}$. Therefore, it is necessary to focus on the problem of mixed outer synchronization.

In recent years, mixed outer synchronization has captured considerable attention of many researchers. Wang et al. ${ }^{7}$ studied the mixed outer synchronization between two complex networks with the same topological structure and identical timevarying coupling delay. Later, Zheng and Shao ${ }^{17}$ analyzed the mixed outer synchronization between two complex networks with non-identical nodes and output couplings by using impulsive hybrid control. Sheng et al. ${ }^{18}$ investigated the mixed outer synchronization between two complex networks with nontime-delayed and time-delayed coupling via pinning control and impulsive control. For the study of finite-time mixed outer synchronization, we refer to Refs. 19-21. Recently, Khan ${ }^{22}$ proposed a scheme for achieving mixed synchronization between two coupled dynamical systems of different dimensions.

In some real complex networks such as telephone communication, stock markets, and brain networks, signals usually travel from one node to another node with lag or delay. Time-delay exists not only in each node of the network, but also in the information transmission between two coupled systems ${ }^{23}$. Thus, it is reasonable to require the states of a response system to synchronize with the past states of the drive system. This kind of synchronization, regarded as lag synchronization, has received considerable attention ${ }^{23-26}$. Recently, Bera et al. ${ }^{27}$ investigated mixed lag synchronization, in which one pair of state variables attains synchronization shifted in time while another pair of state variables is in antisynchronization, however, they are shifted by the same time. Moreover, they observed that when a parameter mismatch is induced in two counter-rotating oscillators, mixed lag synchronization emerges under diffusive coupling via a scalar variable.

In this paper, the lag synchronization behavior between two complex networks will be further developed. More precisely, mixed lag synchronization (MLS), different from that in Ref. 27, is introduced. Here, it means that different state variables of the corresponding nodes can attain lag synchronization, antilag synchronization and even amplitude death simultaneously for a given channel time-delay. In other words, the drive system $x(t)$ and the response system $y(t)$ achieve mixed outer synchronization at a constant time lag, that is, $y(t)=H x(t-\tau)$, where $\tau>0$ and $H=\operatorname{diag}\left(\beta_{1}, \ldots, \beta_{n}\right)\left(\beta_{i} \in\{-1,0,1\}\right)$. This paper is devoted to the MLS between two networks based on hybrid feedback control. The main contribution of this paper includes the following two aspects: (i) Under the controllers based on pinning control with constant feedback gain, we give a sufficient condition which is different from that in the corresponding case ${ }^{18}$. This condition is a simple inequality involving the number of pinned nodes and the coupling strength, which is obtained when the feedback gain is large enough. (ii) To avoid excessive feedback gain, we derive two new conditions for realizing the MLS. One condition is an inequality involving the number of pinned nodes, the coupling strength and the feedback gain, which is obtained under the controllers based on pinning control with constant feedback gain. The other condition is obtained under the controllers based on pinning control with adaptive feedback gain. It is noteworthy that these inequalities can be easily verified. Hence, these two conditions will be widely applied in practice.

\section{MODEL AND PRELIMINARIES}

Some notations are firstly introduced. Let $\mathbb{R}^{n}$ and $\mathbb{R}^{n \times n}$ be the $n$-dimensional Euclidean space and the set of all $n \times n$ real matrices, respectively. $\|\cdot\|$ stands for the Euclidean norm in $\mathbb{R}^{n}$. $A^{\mathrm{T}}$ represents the transpose of matrix $A$. The symbol $\operatorname{diag}\left(a_{1}, \ldots, a_{n}\right)$ means a diagonal matrix with the diagonal elements $a_{1}, \ldots, a_{n} . \quad \mathrm{I}_{m}$ denotes the $m \times m$ identity matrix. $B>0(<0)$ means that matrix $B$ is positive (negative) definite. $\lambda_{\max }(P)$ stands for the largest eigenvalue of matrix $P$.

We consider a complex dynamical network consisting of $N$ dynamical nodes, which is described by:

$$
\begin{aligned}
\dot{x}_{i}(t)= & A_{d} x_{i}(t)+A_{n} x_{i}(t)+f\left(x_{i}(t)\right) \\
& +c \sum_{j=1}^{N} a_{i j} G\left(x_{j}(t)\right), \quad i=1, \ldots, N,
\end{aligned}
$$

where $x_{i}=\left(x_{i 1}, \ldots, x_{i n}\right)^{\mathrm{T}} \in \mathbb{R}^{n}$ is the state vector of the $i$-th node, $A_{d}$ and $A_{n}$ represent the diagonal and non-diagonal matrices, respectively. $f: \mathbb{R}^{n} \rightarrow \mathbb{R}^{n}$ is a smooth nonlinear vector function such that $H f(x)=f(H x)$, where $H$ is a scaling matrix defined 
as:

$$
H=\operatorname{diag}\left(\beta_{1}, \ldots, \beta_{n}\right) \quad\left(\beta_{i} \in\{-1,0,1\}\right) .
$$

$G\left(x_{i}(t)\right)=\left(g\left(x_{i 1}(t), \ldots, g\left(x_{i n}(t)\right)\right)^{\mathrm{T}}\right.$ is a nonlinear vector-valued function, and $c>0$ is the coupling strength. $A=\left(a_{i j}\right)_{N \times N}$ denotes the coupling configuration matrix representing the topological structure of the network, where $a_{i j}$ is defined as follows: if there is a connection from node $j$ to node $i(j \neq i)$, then $a_{i j}>0$; otherwise, $a_{i j}=0$. The diagonal elements of matrix $A$ are defined as $a_{i i}=-\sum_{j=1, j \neq i}^{N} a_{i j}$, $i=1, \ldots, N$.

Remark 1 For the isolated node $i$, the function representing the dynamics is directly written into $A_{d} x_{i}(t)+A_{n} x_{i}(t)+f\left(x_{i}(t)\right)$. In fact, there are many general chaotic systems, such as original Chua's circuit, modified Chua's circuit and Chua's circuit family, that can be written into this form. This will provide great convenience for the design of controllers.

In order to realize MLS between two complex networks, we refer to Network (1) as the drive network, and the response network is given by the following equations:

$$
\begin{aligned}
\dot{y}_{i}(t)= & A_{d} y_{i}(t)+A_{n} y_{i}(t)+f\left(y_{i}(t)\right)+u_{i}(t) \\
& +c \sum_{j=1}^{N} a_{i j} G\left(y_{j}(t)\right), \quad i=1, \ldots, N,
\end{aligned}
$$

where $y_{i}=\left(y_{i 1}, \ldots, y_{i n}\right)^{\mathrm{T}} \in \mathbb{R}^{n}$ is the response state vector of the $i$-th node, $u_{i}$ is a controller to be designed for node $i$, and other notations have the same meanings as those in (1).

Let $\tau$ be a positive time delay. We define the MLS error signal as

$$
e_{i}(t)=y_{i}(t)-H x_{i}(t-\tau), \quad i=1, \ldots, N .
$$

To prove our main results, we need the following definitions, assumptions and lemmas.

Definition 1 The drive network (1) and the response network (2) achieve MLS with time lag $\tau$ with respect to the scaling matrix $H$ if there exists a controller $u_{i}(t)(i=1, \ldots, N)$ for node $i$ such that $\lim _{t \rightarrow \infty}\left\|e_{i}(t)\right\|=0$.

Remark 2 Notice that LS and MOS are two special cases of MLS. In fact, when $H=\mathrm{I}_{N}$, MLS reduces to LS; when $\tau=0$, MLS reduces to MOS.
Definition 2 The drive network (1) and the response network (2) achieve exponential mixed lag synchronization (EMLS) with time lag $\tau$ with respect to the scaling matrix $H$ if there exists a controller $u_{i}(t)(i=1, \ldots, N)$ for node $i$ such that $\left\|e_{i}(t)\right\| \leqslant M e^{-\theta t}$, where $M$ and $\theta$ are two positive constants.

Assumption 1 The nonlinear function $f(x)$ satisfies the Lipschitz condition, i.e., there exists a positive constant $\delta$ such that

$$
\|f(x)-f(y)\| \leqslant \delta\|x-y\|, \quad \forall x, y \in \mathbb{R}^{n} .
$$

Assumption 2 The nonlinear function $G(x)$ satisfies $H G(x)=G(H x)$ and the following inequality

$$
\alpha \leqslant \frac{G(x)-G(y)}{x-y} \leqslant \beta, \quad \forall x, y \in \mathbb{R}^{n} \text { and } x \neq y,
$$

where $\alpha$ and $\beta$ are two positive constants.

Lemma 1 [Refs. 1,28] Let $B=\left(\begin{array}{cc}B_{1} & B_{3} \\ B_{3}^{\mathrm{T}} & B_{2}\end{array}\right)$ and $D=$ $\left(\begin{array}{cc}D_{1} & 0 \\ 0 & 0\end{array}\right)$ be two matrices in $\mathbb{R}^{N \times N}$, where $B_{1}, D_{1} \in$ $\mathbb{R}^{r \times r}(1 \leqslant r<N), \quad B_{1}^{\mathrm{T}}=B_{1}, B_{2}^{\mathrm{T}}=B_{2}$, and $D_{1}=$ $\operatorname{diag}\left(d_{1}, \ldots, d_{r}\right)$ is a positive diagonal matrix. Let $d=\min \left\{d_{1}, \ldots, d_{r}\right\}$. Assume that $d$ is large enough. If $\lambda_{\max }(B-D)$ exists, then we have $\lambda_{\max }(B-D)=$ $\lambda_{\text {max }}\left(B_{2}\right)$.

This result is derived under the condition that $d$ must be large enough. However, it is impractical to assume that $d$ is large enough. To solve this problem, Zhao et al. ${ }^{28}$ further discussed the result of Lemma 1 by introducing a relaxation factor $\varepsilon_{0}$.

Lemma 2 [Ref. 28] If $\lambda_{\max }\left(B_{2}\right)<0$, then for any $\varepsilon_{0}$ $\left(0<\varepsilon_{0}<\left|\lambda_{\max }\left(B_{2}\right)\right|\right)$, there exists a $d>0$ such that $\lambda_{\max }(B-D) \leqslant \lambda_{\max }\left(B_{2}\right)+\varepsilon_{0}<0$.

\section{SYNCHRONIZATION ANALYSIS}

In this section, some synchronization criteria for realizing MLS between two networks will be presented with two kinds of hybrid feedback schemes.

\section{MLS based on pinning control with constant feedback gain}

In this subsection, the MLS between two networks is considered under nonlinear and pinning control with constant feedback gain. Without loss of generality, assume that the first $l(1 \leqslant l<N)$ nodes 
in (2) are controlled. The hybrid feedback control is described as follows:

$u_{i}(t)=u_{1 i}(t)+H A_{n} x_{i}(t-\tau)-A_{n} y_{i}(t), i=1, \ldots, N$

where

$$
u_{1 i}(t)= \begin{cases}-c d e_{i}(t), & i=1, \ldots, l \\ 0, & i=l+1, \ldots, N\end{cases}
$$

Here, $d>0$ denotes the constant feedback control gain.

The error system can be described as

$$
\left\{\begin{aligned}
\dot{e}_{i}(t)= & A_{d} e_{i}(t)+f\left(y_{i}(t)\right)-H f\left(x_{i}(t-\tau)\right) \\
& +c \sum_{j=1}^{N} a_{i j}\left(G\left(y_{j}(t)\right)-H G\left(x_{j}(t-\tau)\right)\right) \\
& -c d e_{i}(t), \quad i=1, \ldots, l, \\
\dot{e}_{i}(t)= & A_{d} e_{i}(t)+f\left(y_{i}(t)\right)-H f\left(x_{i}(t-\tau)\right) \\
& +c \sum_{j=1}^{N} a_{i j}\left(G\left(y_{j}(t)\right)-H G\left(x_{j}(t-\tau)\right)\right), \\
& i=l+1, \ldots, N .
\end{aligned}\right.
$$

Let $A_{1}$ be a modified matrix of $A$ by replacing the diagonal element $a_{i i}$ with $\frac{\alpha}{\beta} a_{i i}$, and let $\tilde{A}=$ $\frac{1}{2}\left(A_{1}+A_{1}^{\mathrm{T}}\right) . \quad \lambda_{l+1}$ denotes the largest eigenvalue of $M_{l+1}$, where $M_{l+1}$ is the minor matrix of $\tilde{A}$ by removing the first $l$ rows and columns. Let $\gamma=$ $\lambda_{\max }\left(A_{d}\right)$. We have the following synchronization criterion.

Theorem 1 Suppose that Assumption 1 and Assumption 2 hold. Let $d$ be large enough. If the number of pinned nodes $l(1 \leqslant l<N)$ satisfies $\lambda_{l+1}<-(\delta+$ $\gamma) /(c \beta)$, then the drive network (1) and the response network (2) can achieve MLS with the feedback control (3).

Proof: We consider the following Lyapunov function

$$
V(t)=\frac{1}{2} \sum_{i=1}^{N} e_{i}^{\mathrm{T}}(t) e_{i}(t)
$$

The derivative of $V(t)$ along the error dynamics (4) is

$$
\begin{aligned}
\dot{V}(t)= & \sum_{i=1}^{N} e_{i}^{\mathrm{T}} e_{i} \\
= & \sum_{i=1}^{N} e_{i}^{\mathrm{T}}\left[A_{d} e_{i}+f\left(y_{i}(t)\right)-H f\left(x_{i}(t-\tau)\right)\right] \\
& +c \sum_{i=1}^{N} \sum_{j=1}^{N} a_{i j} e_{i}^{\mathrm{T}}\left[G\left(y_{j}(t)\right)-H G\left(x_{j}(t-\tau)\right)\right]-c \sum_{i=1}^{l} d e_{i}^{\mathrm{T}} e_{i} \\
= & \sum_{i=1}^{N} e_{i}^{\mathrm{T}}\left[A_{d} e_{i}+f\left(y_{i}(t)\right)-H f\left(x_{i}(t-\tau)\right)\right] \\
& +c \sum_{i=1}^{N} \sum_{j=1, j \neq i}^{N} a_{i j} e_{i}^{\mathrm{T}}\left[G\left(y_{j}(t)\right)-H G\left(x_{j}(t-\tau)\right)\right] \\
& +c \sum_{i=1}^{N} a_{i i} e_{i}^{\mathrm{T}}\left[G\left(y_{i}(t)\right)-H G\left(x_{i}(t-\tau)\right)\right]-c \sum_{i=1}^{l} d e_{i}^{\mathrm{T}} e_{i} .
\end{aligned}
$$

With the assumptions on $f$ and $G$, we get

$$
\begin{aligned}
\dot{V}(t) \leqslant & \sum_{i=1}^{N} e_{i}^{\mathrm{T}} A_{d} e_{i}+\delta \sum_{i=1}^{N} e_{i}^{\mathrm{T}} e_{i} \\
& +c \sum_{i=1}^{N} \sum_{j=1, j \neq i}^{N} a_{i j}\left\|e_{i}^{\mathrm{T}}\right\|\left\|G\left(y_{j}(t)\right)-G\left(H x_{j}(t-\tau)\right)\right\| \\
& +c \sum_{i=1}^{N} a_{i i} e_{i}^{\mathrm{T}}\left[G\left(y_{i}(t)\right)-G\left(H x_{i}(t-\tau)\right)\right]-c \sum_{i=1}^{l} d e_{i}^{\mathrm{T}} e_{i} \\
\leqslant & \sum_{i=1}^{N} e_{i}^{\mathrm{T}} A_{d} e_{i}+\delta \sum_{i=1}^{N} e_{i}^{\mathrm{T}} e_{i}+c \beta \sum_{i=1}^{N} \sum_{j=1, j \neq i}^{N} a_{i j}\left\|e_{i}^{\mathrm{T}}\right\|\left\|e_{j}\right\| \\
& +c \alpha \sum_{i=1}^{N} a_{i i} e_{i}^{\mathrm{T}} e_{i}-c \sum_{i=1}^{l} d e_{i}^{\mathrm{T}} e_{i} \\
\leqslant & e^{\mathrm{T}}\left[(\delta+\gamma) \mathrm{I}_{N}+c \beta\left(\tilde{A}-\frac{D}{\beta}\right)\right] e,
\end{aligned}
$$

where $e=\left(\left\|e_{1}\right\|, \ldots,\left\|e_{N}\right\|\right)^{\mathrm{T}}$ and $D=$ $\operatorname{diag}\{\underbrace{d, \ldots, d}_{l}, \underbrace{0, \ldots, 0}_{N-l}\}$. According to Lemma 1, if $d$ is large enough, we have

$$
\delta+\gamma+c \beta \lambda_{\max }\left(\tilde{A}-\frac{D}{\beta}\right)=\delta+\gamma+c \beta \lambda_{l+1} .
$$

Together with $\lambda_{l+1}<-(\delta+\gamma) /(c \beta)$, we obtain $\dot{V}(t)<0$ which implies $\lim _{t \rightarrow \infty}\left\|e_{i}(t)\right\|=0(i=$ $1, \ldots, N)$. The proof is completed.

Corollary 1 Suppose that Assumption 1 and Assumption 2 hold. Let $d$ be large enough. For some constant $\varepsilon>0$, if the number of pinned nodes $l(1 \leqslant l<N)$ satisfies

$$
\lambda_{l+1}<-\frac{\delta+\gamma+\varepsilon}{c \beta},
$$

then the drive network (1) and the response network (2) can achieve EMLS with the feedback control (3). 
In fact, from the proof of Theorem 1, we derive

$$
\dot{V} \leqslant\left(\delta+\gamma+c \beta \lambda_{l+1}\right) \sum_{i=1}^{N} e_{i}^{\mathrm{T}} e_{i}<-2 \varepsilon V .
$$

According to Comparison lemma ${ }^{29}$, we get $V(t) \leqslant$ $M e^{-2 \varepsilon t}$, where $M=V\left(t_{0}\right) e^{2 \varepsilon t_{0}}$. Furthermore, we get $\left\|e_{i}\right\| \leqslant \sqrt{2 M} e^{-\varepsilon t}$. Thus, two networks (1) and (2) achieve EMLS.

By Theorem 1, we obtain a simple relationship between the number of pinned nodes $l$ and the coupling strength $c$, which can guarantee the MLS between the drive system (1) and the response system (2). To decrease the number of pinned nodes $l$, we can choose a larger coupling strength. It is worth noticing that this result holds under the condition that the feedback gain $d$ must be large enough. However, in engineering applications, a large feedback gain usually causes the system to destabilize and may generate turbulence ${ }^{26}$. To solve this problem, we will use Lemma 2 to further analyze the result of Theorem 1.

Corollary 2 Suppose that Assumption 1 and Assumption 2 hold. For some constant $\varepsilon_{0}$, if there exist $l$ and $d$ such that

$$
\lambda_{\max }\left(\tilde{A}-\frac{D}{\beta}\right) \leqslant \lambda_{l+1}+\varepsilon_{0}<-\frac{\delta+\gamma}{c \beta},
$$

then the drive network (1) and the response network (2) can achieve EMLS with the feedback control (3).

According to Lemma 2, there exist $l$ and $d$ such that $\lambda_{\max }\left(\tilde{A}-\frac{D}{\beta}\right) \leqslant \lambda_{l+1}+\varepsilon_{0}<0$. Thus,

$$
\begin{aligned}
\dot{V} & \leqslant\left[\delta+\gamma+c \beta \lambda_{\max }\left(\tilde{A}-\frac{D}{\beta}\right)\right] \sum_{i=1}^{N} e_{i}^{\mathrm{T}} e_{i} \\
& \leqslant 2\left[\delta+\gamma+c \beta\left(\lambda_{l+1}+\varepsilon_{0}\right)\right] V=-2 q V,
\end{aligned}
$$

where $q=-\left[\delta+\gamma+c \beta\left(\lambda_{l+1}+\varepsilon_{0}\right)\right]>0$. Following the argument of Corollary 1 , we obtain that two networks (1) and (2) achieve EMLS.

Remark 3 To realize MLS between (1) and (2), Corollary 2 gives a sufficient condition which is a simple inequality involving the number of pinned nodes $l$, the coupling strength $c$ and the feedback gain $d$. Hence, it can be easily verified in some applications. Moreover, when $c$ is fixed, we can obtain the smallest value of $l$ and the smallest value of $d$. In fact, since $\lambda_{l+1}$ basically decreases with increasing $l$ (see Fig. 1), we get the smallest value of

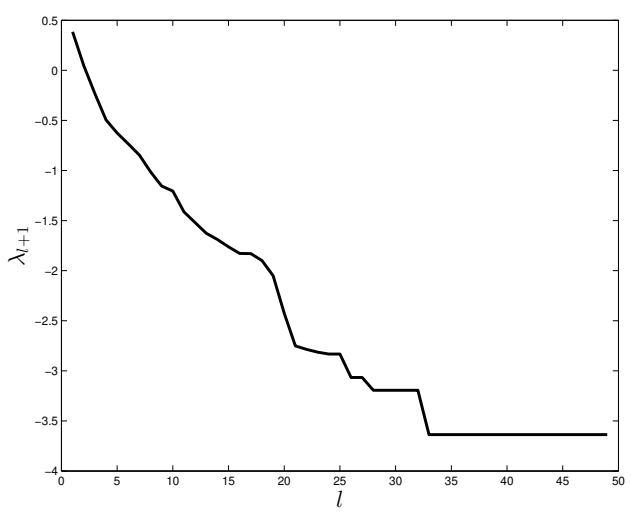

Fig. $1 \lambda_{l+1}$ with respect to the number of pinned nodes $l$.
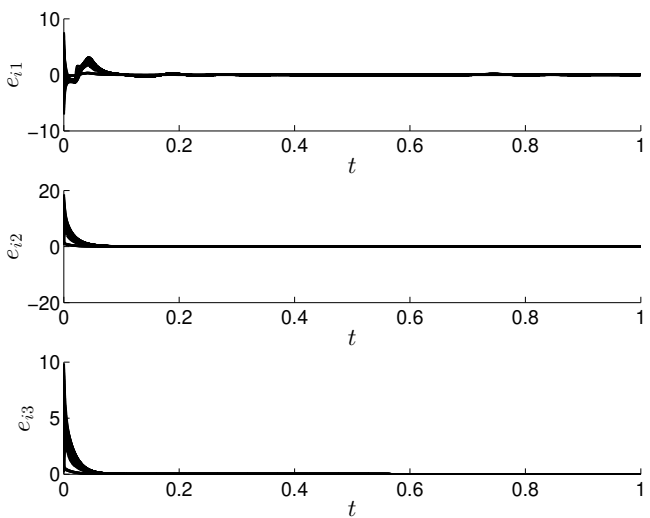

Fig. 2 Synchronization errors with the feedback control (3) when $d=800$.

$l$ (denoted by $l_{\min }$ ) from the right-hand side of (5). Notice that $\lambda_{\max }\left(\tilde{A}-\frac{D}{\beta}\right)$ decreases with increasing $d$ (see Fig. 2). When $l=l_{\min }$, we derive the smallest value of $d$ from the left-hand side of (5).

\section{MLS based on pinning control with adaptive feedback gain}

In order to avoid excessive feedback gains, the MLS between two networks is investigated under nonlinear and pinning control with adaptive feedback gain, which is described as follows:

$u_{i}(t)=u_{1 i}(t)+H A_{n} x_{i}(t-\tau)-A_{n} y_{i}(t), i=1, \ldots, N$,

where

$$
u_{1 i}(t)= \begin{cases}-c d_{i}(t) e_{i}, & i=1, \ldots, l, \\ \dot{d}_{i}(t)=k_{i} e_{i}^{\mathrm{T}} e_{i}, & i=1, \ldots, l, \\ 0, & i=l+1, \ldots, N .\end{cases}
$$


Here, $k_{i}$ is a positive constant.

Theorem 2 Suppose that Assumption 1 and Assumption 2 hold. If there exist the number of pinned nodes $l(1 \leqslant l<N)$ and a positive constant $d^{*}$ such that

$$
(\delta+\gamma) \mathrm{I}_{N}+c \beta\left(\tilde{A}-\frac{D^{*}}{\beta}\right)<0,
$$

where $D^{*}=\operatorname{diag}\{\underbrace{d^{*}, \ldots, d^{*}}_{l}, \underbrace{0, \ldots, 0}_{N-l}\}$, then the drive network (1) and the response network (2) can achieve MLS with the adaptive feedback control (6).

Proof: Construct the following Lyapunov function

$$
V(t)=\frac{1}{2} \sum_{i=1}^{N} e_{i}^{\mathrm{T}}(t) e_{i}(t)+\frac{1}{2} \sum_{i=1}^{l} \frac{c}{k_{i}}\left(d_{i}-d^{*}\right)^{2},
$$

where $d^{*}$ is a positive constant to be determined.

The derivative of $V(t)$ along the error dynamics (4) is

$$
\begin{aligned}
& \dot{V}(t)=\sum_{i=1}^{N} e_{i}^{\mathrm{T}} \dot{e}_{i}+c \sum_{i=1}^{l}\left(d_{i}-d^{*}\right) e_{i}^{\mathrm{T}} e_{i} \\
& =\sum_{i=1}^{N} e_{i}^{\mathrm{T}}\left[A_{d} e_{i}+f\left(y_{i}(t)\right)-H f\left(x_{i}(t-\tau)\right)\right] \\
& +c \sum_{i=1}^{N} \sum_{j=1}^{N} a_{i j} e_{i}^{\mathrm{T}}\left[G\left(y_{j}(t)\right)-H G\left(x_{j}(t-\tau)\right)\right] \\
& -c \sum_{i=1}^{l} d_{i} e_{i}^{\mathrm{T}} e_{i}+c \sum_{i=1}^{l}\left(d_{i}-d^{*}\right) e_{i}^{\mathrm{T}} e_{i} \\
& \leqslant \sum_{i=1}^{N} e_{i}^{\mathrm{T}} A_{d} e_{i}+\delta \sum_{i=1}^{N} e_{i}^{\mathrm{T}} e_{i} \\
& +c \sum_{i=1}^{N} \sum_{j=1, j \neq i}^{N} a_{i j} e_{i}^{\mathrm{T}}\left[G\left(y_{j}(t)\right)-H G\left(x_{j}(t-\tau)\right)\right] \\
& +c \sum_{i=1}^{N} a_{i i} e_{i}^{\mathrm{T}}\left[G\left(y_{i}(t)\right)-H G\left(x_{i}(t-\tau)\right)\right]-c \sum_{i=1}^{l} d^{*} e_{i}^{\mathrm{T}} e_{i} \\
& \leqslant \sum_{i=1}^{N} e_{i}^{\mathrm{T}} A_{d} e_{i}+\delta \sum_{i=1}^{N} e_{i}^{\mathrm{T}} e_{i} \\
& +c \sum_{i=1}^{N} \sum_{j=1, j \neq i}^{N} a_{i j}\left\|e_{i}^{\mathrm{T}}\right\|\left\|\left(G\left(y_{j}(t)\right)-H G\left(x_{j}(t-\tau)\right)\right)\right\| \\
& +c \sum_{i=1}^{N} a_{i i} e_{i}^{\mathrm{T}}\left[G\left(y_{i}(t)\right)-H G\left(x_{i}(t-\tau)\right)\right]-c \sum_{i=1}^{l} d^{*} e_{i}^{\mathrm{T}} e_{i} \\
& \leqslant \sum_{i=1}^{N} e_{i}^{\mathrm{T}} A_{d} e_{i}+\delta \sum_{i=1}^{N} e_{i}^{\mathrm{T}} e_{i}+c \beta \sum_{i=1}^{N} \sum_{j=1, j \neq i}^{N} a_{i j}\left\|e_{i}^{\mathrm{T}}\right\|\left\|e_{j}\right\| \\
& +c \alpha \sum_{i=1}^{N} a_{i i} e_{i}^{\mathrm{T}} e_{i}-c \sum_{i=1}^{l} d^{*} e_{i}^{\mathrm{T}} e_{i} \\
& \leqslant e^{\mathrm{T}}\left[(\delta+\gamma) \mathrm{I}_{N}+c \beta\left(\tilde{A}-\frac{D^{*}}{\beta}\right)\right] e .
\end{aligned}
$$

If there exists $d^{*}>0$ such that $(\delta+\gamma) \mathrm{I}_{N}+$ $c \beta\left(\tilde{A}-\frac{D^{*}}{\beta}\right)<0$, then we get $\dot{V}(t) \leqslant 0$.

Let $E=\{e(t) \mid \dot{V}(t)=0\}$ and $S$ be the largest invariant set in $E$. According to the LaSalle's invariance principle, every solution starting from arbitrary initial values tends to $S$, in which $e(t)$ equals to 0 . This implies that $\lim _{t \rightarrow \infty}\left\|e_{i}(t)\right\|=0(i=1, \ldots, N)$. This completes the proof of Theorem 2 .

Remark 4 In the above four criteria, the outer coupling configuration matrix $A$ can be nonsymmetric and the inner coupling function $G(x)$ can be nonlinear. Compared to the classical assumptions (e.g. $A$ is symmetric and $G(x)$ is linear), our assumptions are more general. Hence, from a theoretical perspective, our results can be widely applied. In our forthcoming works, we will investigate the specific application of these theoretical results in practice.

\section{NUMERICAL SIMULATIONS}

In this section, some numerical examples are presented to illustrate the effectiveness of our results. We choose a scale-free network as the network topology. The dynamics of each node is taken as the modified Chua's circuit system, which is described as

$$
\left\{\begin{array}{l}
\dot{x}_{1}=p\left(x_{2}-\frac{1}{7}\left(2 x_{1}^{3}-x_{1}\right)\right), \\
\dot{x}_{2}=x_{1}-x_{2}+x_{3}, \\
\dot{x}_{3}=-q x_{2},
\end{array}\right.
$$

where $p, q>0$. This system is rewritten into: $\dot{x}(t)=$ $A_{d} x(t)+A_{n} x(t)+f(x(t))$, where

$$
\begin{array}{rlrl}
x & =\left(\begin{array}{l}
x_{1} \\
x_{2} \\
x_{3}
\end{array}\right), & A_{d}=\left(\begin{array}{ccc}
\frac{p}{7} & 0 & 0 \\
0 & -1 & 0 \\
0 & 0 & 0
\end{array}\right), \\
A_{n}=\left(\begin{array}{ccc}
0 & p & 0 \\
1 & 0 & 1 \\
0 & -q & 0
\end{array}\right), & f(x)=\left(\begin{array}{c}
-\frac{2 p x_{1}^{3}}{7} \\
0 \\
0
\end{array}\right) .
\end{array}
$$

Hence, $\gamma=\lambda_{\max }\left(A_{d}\right)=p / 7$. Let $p=10$ and $q=$ 100/7. According to Fig. 1 in Ref. 7, we have $\left|x_{i}(t)\right|<1.5(i=1,2,3)$ for $t>0$. Furthermore, it follows that $\|f(x)-f(y)\| \leqslant \frac{27 p}{14}\|x-y\|$. This means that $f$ satisfies Assumption 1 with $\delta=27 p / 14$. We rearrange the nodes with decreasing degrees and select the first $l$ nodes as the pinned nodes.

For any $x \in \mathbb{R}$, let $g_{1}(x)=\frac{1}{2} \sin x+\frac{21}{2} x$ and $g_{2}(x)=\frac{16}{81} \sin 3 x \cos x+\frac{308}{27} x$. Obviously, the nonlinear vector-valued function $G_{1}(x)=$ $\left(g_{1}\left(x_{1}\right), \ldots, g_{1}\left(x_{n}\right)\right)$ satisfies Assumption 2 with $\alpha=$ 


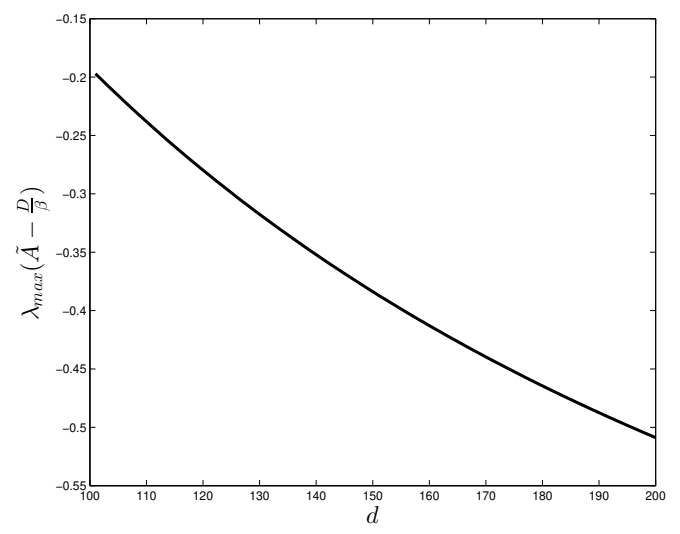

Fig. $3 \lambda_{\max }\left(\tilde{A}-\frac{D}{\beta}\right)$ with respect to varying feedback gain.

10 and $\beta=11$, and the nonlinear vector-valued function $G_{2}(x)=\left(g_{2}\left(x_{1}\right), \ldots, g_{2}\left(x_{n}\right)\right)$ satisfies Assumption 2 with $\alpha=11$ and $\beta=12$.

In what follows, the time lag and the coupling strength are taken as $\tau=0.02$ and $c=8$, respectively. The scaling matrix is given by $H=$ $\operatorname{diag}(1,-1,0)$. Then the error variables are written as:

$$
\begin{aligned}
& e_{i 1}(t)=y_{i 1}(t)-x_{i 1}(t-\tau), \\
& e_{i 2}(t)=y_{i 2}(t)+x_{i 2}(t-\tau), \\
& e_{i 3}(t)=y_{i 3}(t) .
\end{aligned}
$$

For the drive system (1) and the response system (2), the components of initial values are arbitrarily given in the interval $[0,10]$.

\section{MLS with the feedback control (3)}

Example 1 Let $N=50$ and $G(x)=G_{1}(x)$. Fig. 1 shows that $\lambda_{l+1}$ basically decreases with increasing l.

(i) We take $d=800$. By the inequality $\lambda_{l+1}<$ $-(\delta+\gamma) /(c \beta)=-0.2354$, we can obtain $l=$ 4 which is the smallest number of the pinned nodes. The MLS errors are shown in Fig. 2, which implies the correctness of Theorem 1.

(ii) From Corollary 2, we can obtain many feasible combinations of $(l, d)$. Moreover, an appropriate combination can be selected in real situations. For $\varepsilon_{0}=0.777$, by $\lambda_{l+1}+\varepsilon_{0}<$ $-(\delta+\gamma) /(c \beta)$, we obtain $l=8$ which is the smallest number of the pinned nodes. Notice that $\lambda_{\max }\left(\tilde{A}-\frac{D}{\beta}\right)$ decreases with increasing $d$ (see Fig. 3). When $l=8$, we can get $d=124$ which is the smallest feedback gain satisfying
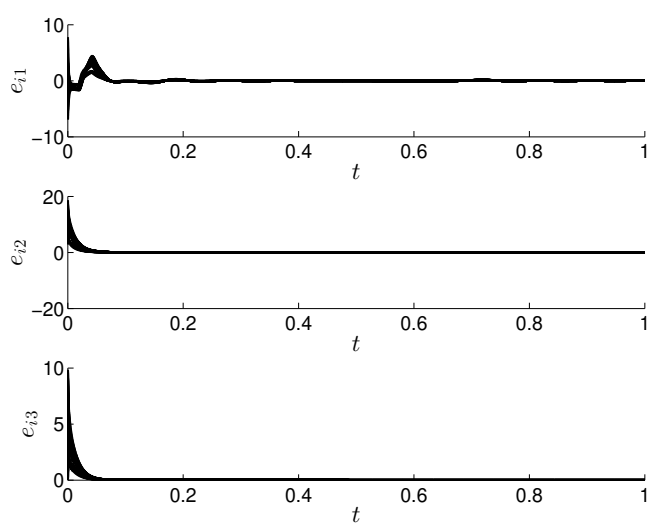

Fig. 4 Synchronization errors with $(l, d)=(8,124)$ for $N=50$ and $G=G_{1}$.
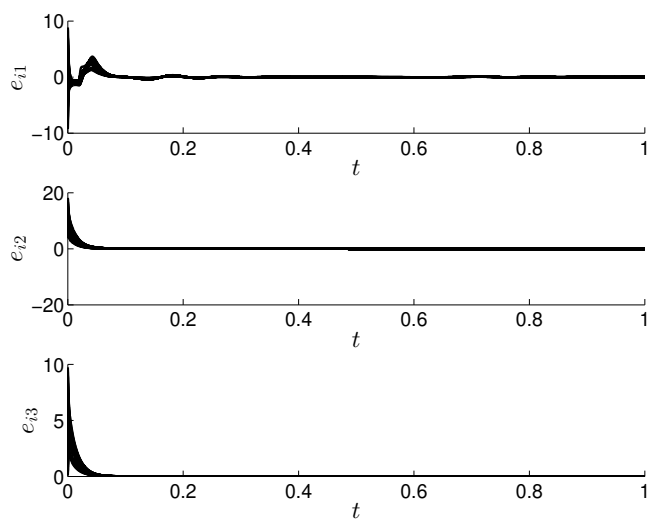

Fig. 5 Synchronization errors with $(l, d)=(8,110)$ for $N=50$ and $G=G_{2}$.

$\lambda_{\max }\left(\tilde{A}-\frac{D}{\beta}\right) \leqslant \lambda_{l+1}+\varepsilon_{0}$. Fig. 4 shows that the EMLS between two networks is realized with $(l, d)=(8,124)$.

Example 2 Let $N=50$ and $G(x)=G_{2}(x)$. According to Corollary 2, we can obtain many feasible combinations of $(l, d)$ and select an appropriate combination. For $\varepsilon_{0}=0.829$, by $\lambda_{l+1}+\varepsilon_{0}<$ $-(\delta+\gamma) /(c \beta)=-0.2158$, we obtain $l=8$ which is the smallest number of the pinned nodes. When $l=8$, we can get the smallest feedback gain $d=110$ satisfying $\lambda_{\max }\left(\tilde{A}-\frac{D}{\beta}\right) \leqslant \lambda_{l+1}+\varepsilon_{0}$. Fig. 5 shows that the EMLS between two networks is realized with $(l, d)=(8,110)$. 

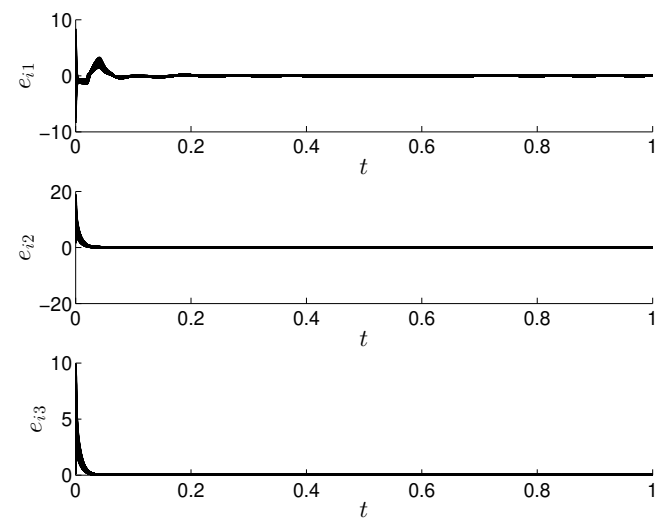

Fig. 6 Synchronization errors with $(l, d)=(18,142)$ for $N=100$ and $G=G_{1}$.
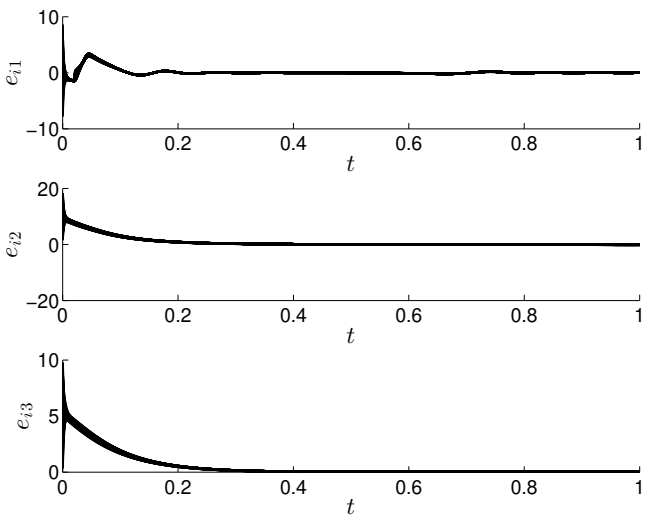

Fig. 7 Synchronization errors with adaptive feedback control (6) for $N=50$ and $G=G_{1}$.

Example 3 Let $N=100$ and $G(x)=G_{1}(x)$. According to Corollary 2 , we can obtain many feasible combinations of $(l, d)$. For $\varepsilon_{0}=3.07$, by $\lambda_{l+1}+$ $\varepsilon_{0}<-(\delta+\gamma) /(c \beta)$, we obtain $l=18$ which is the smallest number of the pinned nodes. When $l=$ 18, we can get the smallest feedback gain $d=142$ satisfying $\lambda_{\max }\left(\tilde{A}-\frac{D}{\beta}\right) \leqslant \lambda_{l+1}+\varepsilon_{0}$. Fig. 6 shows that EMLS between two networks is realized with $(l, d)=(18,142)$.

\section{MLS with the adaptive feedback control (6)}

Example 4 Let $N=50$ and $G(x)=G_{1}(x)$. Some parameters are given as $k_{i}=0.0005$ and $d^{*}=124$. According to $\left[(\delta+\gamma) \mathrm{I}_{N}+c \beta\left(\tilde{A}-\frac{D^{*}}{\beta}\right)\right]<0$, the first 8 nodes can be taken as pinned nodes. The initial value of $d_{i}(t)(i=1, \ldots, 8)$ is arbitrarily given in

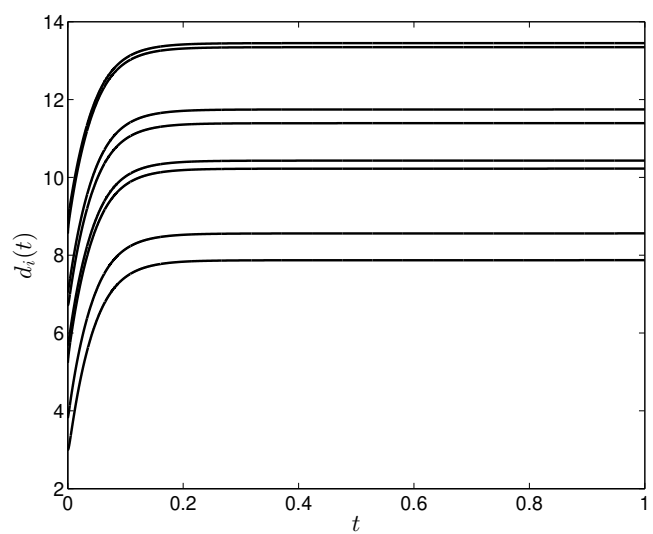

Fig. 8 Evolution of feedback gains for $N=50$ and $G=G_{1}$.
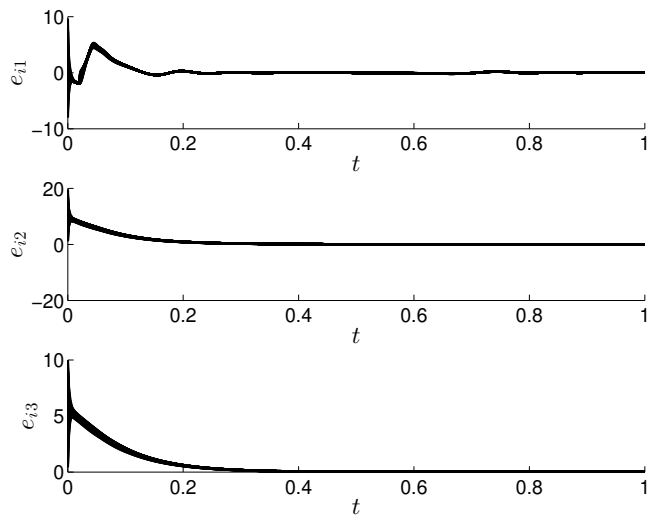

Fig. 9 Synchronization errors with adaptive feedback control (6) for $N=50$ and $G=G_{2}$.

the interval $[0,10]$. Here, $d_{1}(0)=8.5577, d_{2}(0)=$ $6.7080, d_{3}(0)=5.2359, d_{4}(0)=2.9882, d_{5}(0)=$ $7.0397, d_{6}(0)=3.8161, d_{7}(0)=5.6768, d_{8}(0)=$ 8.8786. Fig. 7 shows the MLS errors and Fig. 8 presents the evolution of $d_{i}(t)(i=1, \ldots, 8)$.

Example 5 Let $N=50$ and $G(x)=G_{2}(x)$. Some parameters are given as $k_{i}=0.0005$ and $d^{*}=110$. According to $\left[(\delta+\gamma) \mathrm{I}_{N}+c \beta\left(\tilde{A}-\frac{D^{*}}{\beta}\right)\right]<0$, the first 8 nodes can be taken as the pinned nodes. The initial value of $d_{i}(t)(i=1, \ldots, 8)$ is arbitrarily given in the interval $[0,10]$. Here, $d_{1}(0)=4.5584$, $d_{2}(0)=4.2604, d_{3}(0)=2.1321, d_{4}(0)=1.9325$, $d_{5}(0)=8.3275, d_{6}(0)=7.2664, d_{7}(0)=5.2975$, $d_{8}(0)=8.2908$. Fig. 9 shows the MLS errors and Fig. 10 presents the evolution of $d_{i}(t)(i=1, \ldots, 8)$. 


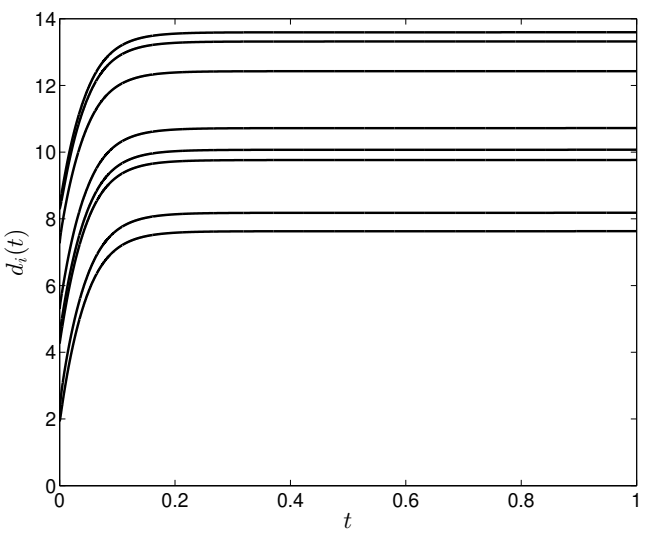

Fig. 10 Evolution of feedback gains for $N=50$ and $G=$ $G_{2}$.
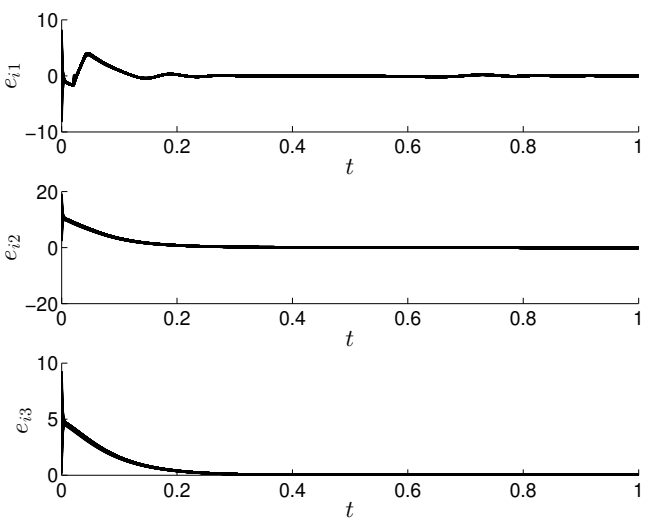

Fig. 11 Synchronization errors with adaptive feedback control (6) for $N=100$ and $G=G_{1}$.

Example 6 Let $N=100$ and $G(x)=G_{1}(x)$. Some parameters are given as $k_{i}=0.0005$ and $d^{*}=142$. According to $\left[(\delta+\gamma) \mathrm{I}_{N}+c \beta\left(\tilde{A}-\frac{D^{*}}{\beta}\right)\right]<0$, the first 18 nodes can be taken as the pinned nodes. The initial value of $d_{i}(t)(i=1, \ldots, 18)$ is arbitrarily given in the interval $[0,10]$. Fig. 11 shows the MLS errors, and Fig. 12 presents the evolution of $d_{i}(t)$ $(i=1, \ldots, 18)$.

For the above three examples, it is obvious that the value of the adaptive feedback gain gets stabilized at some constant. This constant is much smaller than the constant feedback gain of the corresponding case in "MLS with the feedback control (3)". These figures of synchronization errors demonstrate the effectiveness of Theorem 2.

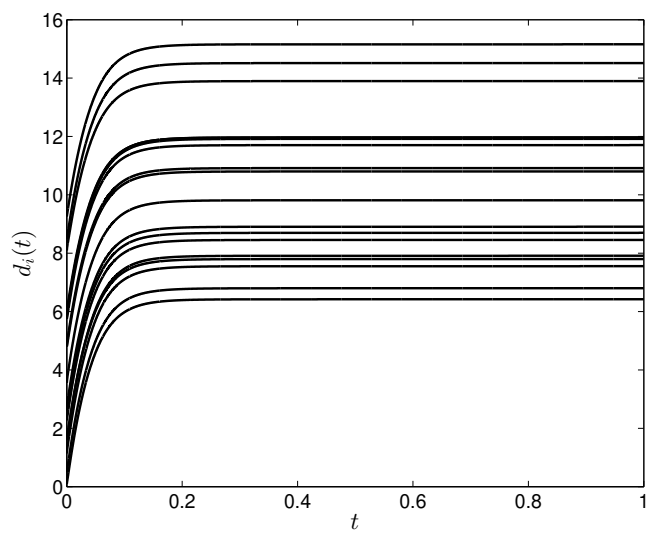

Fig. 12 Evolution of feedback gains for $N=100$ and $G=$ $G_{1}$.

\section{CONCLUSIONS}

In many real situations, time-delay is inevitable. In this paper, we have introduced mixed lag synchronization (MLS), where the response system achieves MOS with the drive system at a constant time lag. By using hybrid feedback control, the MLS between two complex networks has been investigated. Based on pinning control with constant feedback gain, two sufficient conditions for realizing MLS between two networks have been given when the feedback gain is large enough. These conditions are different from the previous one in the corresponding case. To avoid excessive feedback gain, two new conditions have been derived to realize the MLS. One condition is an inequality involving the number of pinned nodes, the coupling strength and the feedback gain, which is obtained under the controllers based on pinning control with constant feedback gain. The other condition is obtained under the controllers based on pinning control with adaptive feedback gain. It is noteworthy that these inequalities can be easily verified. Therefore, these conditions can be easily carried out in some applications. Finally, some numerical examples have been presented to illustrate the effectiveness of the theoretical results.

Acknowledgements: The authors would like to express their sincere gratitude to Professor Xiaoqun Wu (Wuhan University) for her thorough review, helpful suggestions and grammatical corrections. The authors also would like to express their sincere thanks to the editors and the referees for their careful reading and valuable suggestions. This work was supported by the National Natural Science Foundation of China (Grant No. 11401595). 


\section{REFERENCES}

1. Wang XF, Chen RG (2002) Pinning control of scalefree dynamical networks. Phys A 310, 281-2.

2. Fan CX, Jiang GP, Jiang FH (2010) Synchronization between two complex dynamical networks using scalar signals under pinning control. IEEE Trans Circuits Syst 57, 2991-98.

3. Li CP, Sun WG, Kurths J (2007) Synchronization between two coupled complex networks. Phys Rev E 76, 046204.

4. Rulkov NF, Sushchik MM, Tsimring LS, Abarbanel HD (1995) Generalized synchronization of chaos in directionally coupled chaotic systems. Phys Rev E 51, 980-94.

5. Tang HW, Chen L, Lu JA, Tse CK (2008) Adaptive synchronization between two complex networks with nonidentical topological structures. Phys A 387, 5623-30.

6. Wu XQ, Zheng WX, Zhou J (2009) Generalized outer synchronization between complex dynamical networks. Chaos 19, 013109.

7. Wang JW, Ma Q, Zeng L, Abd-Elouahab, M (2011) Mixed outer synchronization of coupled complex networks with time varying coupling delay. Chaos 21, 013121.

8. Grosu I, Banerjee R, Roy PK, Dana SK (2009) Design of coupling for synchronization of chaotic oscillators. Phys Rev E 80, 016212.

9. Wang T, Wang KJ, Ja N (2011) Chaos Control and Hybrid Projective Synchronization of a Novel Chaotic System. Math Probl Eng 2011, 452671.

10. Zhou P, Ding R, Cao YX (2012) Hybrid projective synchronization for two identical fractional-order chaotic systems. Discrete Dyn Nat Soc, 768587.

11. Mainieri R, Rehacek J (1999) Projective synchronization in three-dimensional chaotic systems. Phys Rev Lett 82, 3024-45.

12. Wei Q, Xie CJ, Liang Y, Lin D, Wang B (2017) Hybrid time-varying delay projective synchronization in complex dynamical networks. Trans Institute Meas Control 39, 1339-45.

13. Xing JS (2012) Adaptive Hybrid Function Projective Synchronization of Chaotic Systems with TimeVarying Parameters. Math Probl Eng 2012, 619708.

14. Chee CY, Xu D (2005) Secure digital communication using controlled projective synchronisation of chaos. Chaos Soliton Fract 23, 1063-70.

15. Li, KZ, Zhao, MC, and Fu, XC (2009) Projective synchronization of driving-response systems and its application to secure communication. IEEE Trans Circuits Sys I 56, 2280-91.

16. Song XN, Song S, Li B, Balsera IT (2017) Adaptive projective synchronization for timedelayed fractional-order neural networks with uncertain parameters and its application in secure communications. Trans Institute Meas Control,
014233121771452.

17. Zheng S, Shao W (2013) Mixed outer synchronization of dynamical networks with nonidentical nodes and output coupling. Nonlinear Dynam 73, 2343-52.

18. Sheng S, Feng JW, Tang Z, Zhao Y (2015) Mixed outer synchronization of two coupled complex networks with time-varying delay coupling and non-delay coupling. Nonlinear Dynam 80, 803-15.

19. He P, Ma SH, Fan T (2012) Finite-time mixed outer synchronization of complex networks with coupling time-varying delay. Chaos 22, 043151.

20. Jing TY, Chen FQ, Li QH (2015) Finite-time mixed outer synchronization of complex networks with time-varying delay and unknown parameters. Appl Math Model 39, 7734-43.

21. Tang XY, Yang ZY, Zhang J (2018) Adaptive finitetime mixed interlayer synchronization of two-layer complex networks with time-varying coupling delay. Adv Math Phys 2018, 7025404.

22. Khan MA (2017) Mixed synchronization scheme for coupled different dimensional dynamical systems. Int J Appl Comput Math 3, 2687-94.

23. Yang HL, Shu L, Zhong SM (2017) Pinning lag synchronization of complex dynamical networks with known state time-delay and unknown channel timedelay. Nonlinear Dynam 89, 1793-2.

24. Li ZW, Qiu ZY, Sun WG (2016) Pinning lag synchronization between two dynamical networks with nonderivative and derivative couplings. Kybernetika 52, 648-60.

25. Sun YH, Cao JD (2007) Adaptive lag synchronization of unknown chaotic delayed neural networks with noise perturbation. Phys Lett A 364, 277-85.

26. Wen BH, Zhao M, Meng, FY (2014) Pinning synchronization of the drive and response dynamical networks with lag. Arch Control Sci 24, 257-70.

27. Bera BK, Bhowmick SK, Ghosh D (2017) Mixed lag synchronization in chaotic oscillators and experimental observations. Int J Dynam Control 5, 269-73.

28. Zhao JC, Lu JA, Wu XQ (2010) Pinning control of general complex dynamical networks with optimization. Sci China Inf Sci 53, 813-22.

29. Khalil H (2002) Nonlinear Systems, 3rd edn, Prentice Hall, Englewood Cliffs, New Jersey. 